\title{
ISOMORPHISMS BETWEEN GROUPS OF DIFFEOMORPHISMS
}

\author{
TOMASZ RYBICKI
}

(Communicated by Roe Goodman)

\begin{abstract}
It is known that the group of all diffeomorphisms of a manifold determines uniquely the topological and smooth structure of the manifold itself. We specify a possibly large class of diffeomorphism groups which satisfy this property. In particular, so does the group of contact diffeomorphisms.
\end{abstract}

\section{INTRODUCTION}

In this paper we generalize a theorem of Filipkiewicz [5] stating that the group $\operatorname{Diff}^{r}(M)$ of all $C^{r}$ diffeomorphisms of a manifold $M, 1 \leq r \leq \infty$, defines the smooth structures of $M$. The theorem of Filipkiewicz was proven by using techniques developed in earlier papers of Whittaker [15] and Takens [13]. More recently, Banyaga in [3] obtained analogous theorems for the groups of automorphisms of a volume form structure as well as symplectic structure. All the results constitute an essential contribution to the Erlangen Program of F. Klein.

Our purpose is to define a possibly large class of diffeomorphism groups which satisfy the theorem of Filipkiewicz. In our axiomatization imposed on groups of diffeomorphisms we omit the property of local perfectness of a group. This property plays a crucial role in [3] and, in fact, enables Banyaga to extend the theorem of Filipkiewicz in the case of automorphism groups mentioned above. This method, however, depends essentially on deep theorems of simplicity and perfectness of diffeomorphism groups due to Thurston [14], Mather [8], and Banyaga [1]. On the other hand, the method of Whittaker-Filipkiewicz cannot be directly applied to the case of automorphism groups for the reasons explained in [3]. In the present note we change and simplify the arguments used in [3] and [5] and we get rid of the perfectness property. As an application an analogue of the Filipkiewicz theorem for the group of contact diffeomorphisms is obtained (this has been left as an open problem in [3]).

Let us mention that in another of the author's papers [12] an analogous theorem for a large class of nontransitive groups of diffeomorphisms is shown.

Received by the editors April 16, 1993.

1991 Mathematics Subject Classification. Primary 58D05, 57R50, 22 E65.

Key words and phrases. Automorphism of geometric structure, group of diffeomorphisms, isomorphism of groups, contact structure, Erlangen Program. 
All manifolds are assumed to be $C^{\infty}$ smooth connected boundaryless and second countable.

\section{AXIOMS AND THE MAIN RESUlt}

Let us recall that $\operatorname{Diff}^{r}(M)$ is a topological group with $C^{r}$ topology. Any subgroup $G=G(M)$ of $\operatorname{Diff}^{r}(M)$ will be called a group of diffeomorphisms of $M$. If a geometric structure $\alpha$ of $M$ is under consideration, then a subgroup $G$ of $\operatorname{Diff}^{r}(M, \alpha)$, the group of the $\alpha$-preserving diffeomorphisms, is said to be a group of automorphisms of $(M, \alpha)$.

We introduce some notation. If $f \in \operatorname{Diff}^{r}(M)$ then

$$
\begin{aligned}
\operatorname{supp}(f) & =\overline{\{x \in M \mid f(x) \neq x\}}, \\
\operatorname{Fix}(f) & =\{x \in M \mid f(x)=x\} .
\end{aligned}
$$

The symbol $\operatorname{Diff}^{r}(M)_{\mathrm{c}}$ stands for the group of all compactly supported diffeomorphisms of $\operatorname{Diff}^{r}(M)$, and $G(M)_{\mathrm{c}}=G(M) \cap \operatorname{Diff}^{r}(M)_{\mathrm{c}}$. Next $\operatorname{Diff}^{r}(M)_{0}$ (resp. $G(M)_{0}$ ) is the subgroup of all $C^{r}$ diffeomorphisms $f$ (resp. belonging to $G(M)$ ) for which there exist a compact subset $K$ of $M$ and an isotopy $\left\{f_{t}\right\}$ connecting $f$ with the identity, with $f_{t}$ fixed outside $K$.

For $x \in M, S_{x} G$ denotes the isotropy subgroup of $G$; i.e., $S_{x} G=\{g \in$ $G \mid g(x)=x\}$. Observe that $f S_{x} G f^{-1}=S_{y} G$ whenever $f(x)=y$.

Next for any open $U$ of $M$ by $G(U)_{\text {c }}$ (resp. $\left.G(U)_{0}\right)$ we denote all elements of $G$ compact supported in $U$ (resp. isotopic to the identity with compactly supported isotopy in $U)$.

By $[G, G]$ we denote the commutator subgroup of $G$, i.e., the subgroup generated by commutators $[f, g]=f g f^{-1} g^{-1}$, where $f, g \in G$. Let us recall that $G$ is said to be perfect if $G=[G, G]$.

Recall that $U$ is called an open ball if $U=h\left(K_{1}\right)$ for some embedding $h: K_{1+\varepsilon} \rightarrow M$ with some $\varepsilon>0, K_{r}$ being an open ball in $\mathbf{R}^{n}$ of radius $r$.

Now we wish to introduce some axioms. These seem to be indispensable in order that $G=G(M, \alpha)$, the group of all automorphisms of $(M, \alpha)$, determine the smooth (and geometric) structure of $(M, \alpha)$.

Axiom 1. Let $\mathscr{U}=\left\{U_{j}\right\}$ be any finite open cover. If $f \in G_{0}$, then $f=f_{1} \cdots f_{s}$ and $\operatorname{supp}\left(f_{i}\right) \subset U_{j(i)}$ for $i=1, \ldots, s$.

It is well known that $G=\operatorname{Diff}^{r}(M)_{0}$ satisfies Axioms 1 (cf. Palis and Smale [10]). Also the kernels of the flux homomorphisms of $\operatorname{Diff}(M, \alpha)$, where $\alpha$ is a volume element or a symplectic form, have this property [1].

Axiom 2. For any $x \in U, U$ open, there is $f \in G(U)_{0}$ such that $f(x) \neq x$. Moreover, for any $x \in M$ and a sufficiently small open ball $U$ with a center at $x$ there exists $g \in G_{0}$ with $\operatorname{Fix}(g)=(M-U) \cup\{x\}$. [3]).

Again, this axiom is satisfied by "classical" groups of diffeomorphisms (cf.

Definition. A group of diffeomorphisms is said to have $T(n)$ property if for any pairwise distinct $n$-tuples $x_{1}, \ldots, x_{n} ; y_{1}, \ldots, y_{n} \in M$ there is $f \in G$ such that $f\left(x_{i}\right)=y_{i}$.

Axiom 3. $G$ acts $T(3)$ on $M$. 
In the case of the "classical" groups of a geometric structure automorphisms the following notation is useful.

Definition. A group of diffeomorphisms $G(M)$ satisfies the path transitivity if for any $x, y \in M$ and for any path $c:[0,1] \rightarrow M$ such that $c(0)=x$, $c(1)=y$ there exists $f \in G(M)$ with its support contained in an arbitrarily small neighborhood of $\operatorname{im}(c)$ such that $f(x)=y$.

It is well known that $\operatorname{Diff}^{\gamma}(M)$ possesses this property as well as $\operatorname{Diff}^{\prime}(M, \alpha)$, where $\alpha$ is a volume element or a symplectic form.

Proposition 1. Let $G(M)$ be a group of diffeomorphisms, and let $\operatorname{dim}(M)>1$. Then the path transitivity implies the $T(n)$ property.

The proof is straightforward.

The theorems of Filipkiewicz and Banyaga are an "integral" version of Pursell-Shanks type theorems. A theorem of Pursell-Shanks states that the Lie algebra of vector fields of a manifold $M$ determines completely the smooth structure of $M$ itself [11]. A whole suite of generalizations followed, e.g. [9, 7]. In order to connect these two types of theorems let us introduce the following:

Definition. A Lie algebra of compactly supported vector fields of a manifold $M$ satisfies (PS) property if it determines uniquely the $C^{\infty}$ structure of $M$ (in case of a geometric structure $(M, \alpha)$ one claims the determination of the $\alpha$ too). Moreover, if $\mathscr{X}\left(M_{1}\right), \mathscr{X}\left(M_{1}\right)$ are isomorphic by $\Phi$, then the unique diffeomorphism $\phi: M_{1} \rightarrow M_{2}$ fulfills $\Phi=\phi_{*}$.

Now our last axiom will appeal to a better known structure of a Lie algebra of vector fields rather than to a group of diffeomorphisms. On the other hand, a close investigation of the Whittaker-Takens-Filipkiewicz method leads to a conclusion that it cannot be reduced to the infinitesimal case. As Pursell-Shanks type theorems have been proved in the $C^{\infty}$ category, we will assume that in the following axiom $G$ is a group of $C^{\infty}$ diffeomorphisms.

Axiom 4. There is $\mathscr{Z}$, a Lie algebra of vector fields satisfying (PS) property, such that all elements of the one-parameter transformation group of any element of $\mathscr{X}$ belong to $G$.

Now our main result can be formulated as follows.

Theorem 1. Let $M_{i}, i=1,2$, be two manifolds, possibly equipped with geometric structures $\alpha_{i}$. Suppose $G\left(M_{i}\right)$ are diffeomorphism groups of $M_{i}$, satisfying Axioms 1, 2, and 3. If there is a group isomorphism $\Phi: G\left(M_{1}\right) \rightarrow G\left(M_{2}\right)$, then there is a unique homeomorphism $\phi: M_{1} \rightarrow M_{2}$ such that $\Phi(f)=\phi f \phi^{-1}$ for any $f \in G\left(M_{1}\right)$. Moreover, if Axiom 4 is satisfied, then $\phi$ is a diffeomorphism (and an isomorphism of $\left(M_{1}, \alpha_{1}\right)$ onto $\left(M_{2}, \alpha_{2}\right)$ ).

As an important consequence we have

Corollary 1. If $G(M)$ satisfies Axioms $1,2,3$, and 4 , then every automorphism of $G(M)$ is inner.

Proof of the second assertion (see also [2]). Assume that there is a homeomorphism $\phi: M_{1} \rightarrow M_{2}$ such that $\Phi(f)=\phi f \phi^{-1}$ for any $f \in G\left(M_{1}\right)$. We wish to define a Lie algebra isomorphism $\Psi: \mathscr{X}\left(M_{1}\right) \rightarrow \mathscr{X}\left(M_{2}\right)$, where $\mathscr{X}\left(M_{i}\right)$ is 
a Lie algebra of vector fields on $M_{i}$ satisfying Axiom 4 for the automorphism group $G\left(M_{i}, \alpha_{i}\right)$. By (PS) property this will complete the proof. In fact, $\Psi$ induces a diffeomorphism $\psi: M_{1} \rightarrow M_{2}$ satisfying $\psi_{*}=\Psi$. On the other hand, the definition of $\Psi$ below implies $\phi_{*}=\Psi$. This $\phi=\psi$ and $\phi$ is a diffeomorphism.

Let $X \in \chi\left(M_{1}\right)$, and let $g_{t}$ be its one-parameter group of diffeomorphisms. Consider $h_{t}=\phi g_{t} \phi^{-1}$. By the assumption the $h_{t}$ 's are $C^{\infty}$ diffeomorphisms. They obviously satisfy $h_{t+s}=h_{t} h_{s}$ and $h_{0}=\mathrm{id}_{M_{2}}$. Moreover the action of $\mathbf{R}$ on $M_{2}$ by $(t, x) \rightarrow h_{t}(x)$ is clearly a continuous action by diffeomorphisms, so that, in view of a well-known theorem of Montgomery and Zippin, it is $C^{\infty}$ smooth in both variables. Therefore, it is a one-parameter transformation group of a vector field $\Psi(X)$ belonging, by the definition and the axiom, to $G\left(M_{2}\right)$.

By the first part of the theorem, the group isomorphism $\Phi^{-1}$ must determine the homeomorphism $\phi^{-1}$. Consequently, the respective mapping from $\chi_{2}$ to $\chi_{1}$ must be the inverse of $\Psi$. Thus $\Psi$ is a bijection.

It remains to prove that $\Psi$ is a Lie algebra homomorphism. In fact, due to the equality

$$
(\Psi(X) f) \circ \phi=X(f \circ \phi),
$$

where $X \in \chi_{1}, f \in C^{\infty}\left(M_{2}\right)$, one has

$$
\begin{aligned}
(\Psi[X, Y] f) \circ \phi & =[X, Y](f \circ \phi) \\
& =X((\Psi(Y) f) \circ \phi)-Y((\Psi(X) f) \circ \phi) \\
& =\Psi(X)(\Psi(Y)(f)) \circ \phi-\Psi(Y)(\Psi(X)(f)) \circ \phi \\
& =([\Psi(X), \Psi(Y)] f) \circ \phi,
\end{aligned}
$$

and

$$
\Psi[X, Y]=[\Psi(X), \Psi(Y)]
$$

This completes the proof.

\section{THE PROOF OF THEOREM 1}

We adopt once and for all the notation from Theorem 1: $\Phi: G\left(M_{1}\right) \rightarrow G\left(M_{2}\right)$ is a group isomorphism. In this section we will assume that all the axioms are satisfied (nevertheless some facts presented below do not require all the axioms). We begin with the following two lemmas proved by Whittaker (Lemmas 6 and 8 in [15]). They give an important characterization of the isotropy subgroups and will play a central role in the sequel. The proofs in our case are identical and will be omitted (for the proofs see also Filipkiewicz [5]).

Lemma 1. Assume that $G=G(M)$, a group of diffeomorphisms of $M$, satisfies Axiom 3. Let $x \in M, S_{x}=S_{x} G$ the isotropy subgroup at $x, f, g, h \in G-S_{x}$. Then (a) $G-S_{x}=\left(S_{x} f S_{x}\right) \cup\left(S_{x} f S_{x}\right)^{-1}$; (b) $g, h \in S_{x} f S_{x}$ implies that $s_{1} s_{2}=t_{2} t_{1}$ for some $s_{1}, t_{1} \in f^{-1} S_{x} g \cap S_{x}$ and $s_{2}, t_{2} \in f^{-1} S_{x} h \cap S_{x}$.

As an obvious consequence of (a) we have

Corollary 2. $S_{x} G$ is a maximal subgroup of $G$.

Lemma 2. Let $F=\Phi^{-1}\left(S_{y} G\left(M_{2}\right)\right)$ for some $y \in M_{2}$, and let $A$ be a closed proper nonempty subset of $M_{1}$. If $f(A)=A$ for any $f \in F$, then $A=\{x\}$ and $F=S_{x} G\left(M_{1}\right)$ for some $x \in M_{1}$. 
The idea that lies behind the proof of Theorem 1 is to show that the isotropy subgroups $S_{x} G\left(M_{1}\right)$ are mapped by $\Phi$ onto $S_{y} G\left(M_{2}\right)$ and vice versa. This property implies immediately Theorem 1 due to the following:

Proposition 2 ([5, 3]). Let $\Phi: G\left(M_{1}\right) \rightarrow G\left(M_{2}\right)$ satisfy the property: $\forall x \in$ $M_{1} \exists y \in M_{2} \Phi\left(S_{x} G\left(M_{1}\right)\right)=S_{y} G\left(M_{2}\right)$. Then there is a unique homeomorphism $\phi: M_{1} \rightarrow M_{2}$ such that $\Phi(f)=\phi f \phi^{-1}$ for any $f \in G\left(M_{1}\right)$.

Now the remaining part of the proof of Theorem 1 consists in showing that the isomorphism $\Phi$ satisfies the assumption of the proposition. Modifying slightly the method of Filipkiewicz we introduce the following notation. For $y \in M_{2}$, we let $\mathscr{C}_{y} \in \mathscr{B}\left(M_{1}\right)$ be the totallity of $U$ satisfying

$$
G(U)_{0} \subset F_{y}^{1}=\Phi^{-1}\left(S_{y} G\left(M_{2}\right)\right),
$$

where $\mathscr{B}\left(M_{1}\right)$ is some topological base by open balls in $M_{1}$ which is $G\left(M_{1}\right)$ invariant. Similarly we define the set $\mathscr{D}_{x}$ where $x \in M_{1}$, as the set of open balls from a topological $G\left(M_{2}\right)$-invariant base $\mathscr{B}\left(M_{2}\right)$ such that

$$
G(V)_{0} \subset F_{x}^{2}=\Phi\left(S_{x} G\left(M_{1}\right)\right) \text {. }
$$

Let $C_{y}=M_{1}-\bigcup \mathscr{C}_{y}, D_{x}=M_{2}-\bigcup \mathscr{D}_{x}$. Our aim is to show that both $C_{y}$ and $D_{x}$ consist of one element.

Lemma 3. The subsets $C_{y}$ and $D_{x}$ are closed and nonempty. Furthermore, $f\left(C_{y}\right) \subset C_{y} \forall f \in F_{y}^{1}$ and $g\left(D_{x}\right) \subset D_{x} \forall g \in F_{x}^{2}$.

Proof. It is clear that $C_{y}$ and $D_{x}$ are closed. Let $U \in \mathscr{C}_{y}$ and $f \in F_{y}^{1}$. If $V=f(U)$, then $G(V)_{0}=f G(U)_{0} f^{-1} \subset f F_{y}^{1} f^{-1}=F_{y}^{1}$. It follows that $f\left(C_{y}\right) \subset C_{y}$. Similarly $g\left(D_{x}\right) \subset D_{x}$ for any $g \in F_{x}^{2}$.

Finally we show that $C_{y}$ (and similarly $D_{x}$ ) is nonempty. Arguing by contradiction suppose that $C_{y}$ is empty, i.e., $\mathscr{C}_{y}$ is an open cover of $M$ by balls $U_{i}$ such that $G\left(U_{i}\right)_{0} \subset F_{y}$ for each $i$. Then, by Axiom 1, $G\left(M_{1}\right)_{0} \subset F_{y}{ }^{1}$. In fact, for any $f \in G\left(M_{1}\right)_{0}$ one can choose a finite subcover $\left\{U_{i_{k}}\right\}$ of $\mathscr{C}_{y}$ such that $\operatorname{supp}(f) \subset \bigcup_{k} U_{i_{k}}$. Now due to the transitivity of $G\left(M_{2}\right)$ one has that $G\left(M_{1}\right)_{0} \subset F_{z}^{1}$ for any $z \in M_{2}$. Thus we get $G\left(M_{1}\right) \subset \bigcap_{y \in M_{2}} F_{y}^{1}=\{\mathrm{id}\}$, a contradiction.

Lemma 4. If $f, g \in G(M)$ satisfy $f g=g f$, then $f(\operatorname{Fix}(g))=\operatorname{Fix}(g)$.

The proof is straightforward.

The proof of the following fact omits the property of local perfectness and gives an essential simplification of the "traditional" argument.

Proposition 3. For any $y \in M_{2}$ there is an open ball $U$ in $M_{1}$ such that $G(U)_{0} \subset F_{y}^{1}$.

Proof. Let $y \in M_{2}$. For any open ball $V$ in $M_{1}$ we may assume the existence of $f_{1} \in G(V)_{0}$ such that $f_{2}(y) \neq y$ and $f_{2}^{2}(y) \neq y$ where $f_{2}=\Phi\left(f_{1}\right)$; otherwise, we are done. Then take two open balls $V, W$ with $\bar{V} \cap \bar{W}=\varnothing$, with $f_{1} \in G(V)_{0}$ as above, and $g_{1} \in G(W)_{0}$ such that $g_{2}(y) \neq y$ and $g_{2}^{2}(y) \neq y$, where $g_{2}=\Phi\left(g_{1}\right)$.

Next by Axiom 2 we can choose $h_{2} \in G\left(M_{2}\right)_{0}$ such that Fix $\left(h_{2}\right)=\left(M_{2}-B\right) \cup y$ for some open ball $B$ at $y$ and the conditions

$$
B \cap f_{2}(B)=\varnothing, B \cap f_{2}^{2}(B)=\varnothing, B \cap g_{2}(B)=\varnothing, B \cap g_{2}^{2}(B)=\varnothing,
$$


are fulfilled. Let $h_{1}=\Phi^{-1}\left(h_{2}\right)$. The two following cases can occur: either (1) $\overline{V \cup h_{1}(V)} \neq M_{1}$ or $(2) \overline{V \cup h_{1}(V)}=M_{1}$.

In case (1) take $U$ such that $\bar{U} \cap \overline{\left(V \cup h_{1}(V)\right)}=\varnothing$. For any $k_{1} \in G(U)_{0}$ obviously $\left[k_{1}, f_{1} h_{1} f_{1}^{-1} h_{1}^{-1}\right]=\mathrm{id}$ as $\operatorname{supp}\left(f_{1} h_{1} f_{1}^{-1} h_{1}^{-1}\right) \subset V \cup h_{1}(V)$. Therefore, for $k_{2}=\Phi\left(k_{1}\right)$

$$
\left[k_{2}, f_{2} h_{2} f_{2}^{-1} h_{2}^{-1}\right]=\mathrm{id}
$$

and similarly

$$
\left[k_{2}, f_{2}^{2} h_{2} f_{2}^{-2} h_{2}^{-1}\right]=\mathrm{id} .
$$

Then Lemma 4 gives either $k_{2}(y)=y$ or $k_{2}(y)=f_{2}(y)$ by the first equality and either $k_{2}(y)=y$ or $k_{2}(y)=f_{2}^{2}(y)$ by the second one. Consequently one has $k_{2}(y)=y$, since $f_{2}(y) \neq f_{2}^{2}(y)$. Thus $G(U)_{0} \subset F_{y}^{1}$.

In case (2) we get $h_{1}^{-1}(\bar{W}) \subset V$ so that $\overline{W \cup h_{1}(W)} \neq M_{1}$. Thus (1) is satisfied for $W$ and we can proceed as above.

It is evident that Theorem 1 follows by Lemmas 2 and 3 and by Propositions 2 and 3.

\section{Application to CONTACt structures}

Let us recall some basic facts for the global theory of contact structures. The references are $[4,6,9]$.

Let $\omega$ be a contact form on $M$, i.e., $\omega$ is a 1 -form on $M$ such that $\omega \wedge(d \omega)^{n} \neq 0 \quad(\operatorname{dim}(M)=2 n+1)$. A contact diffeomorphism is a diffeomorphism $h: M \rightarrow M$ such that $h^{*} \omega=\lambda \omega$ for some positive function $\lambda$. Let us denote by $G(M, \omega)_{0}$ the connected component of the topological group $G(M, \omega)$ of all contact diffeomorphisms. Then $G(M, \omega)_{0}$ consists of all contact diffeomorphisms compactly isotopic through elements of $G(M, \omega)_{0}$ with the identity. Next by $\chi_{\omega}(M)$ we denote the Lie algebra of all contact vector fields, i.e., $X \in \chi_{\omega}(M)$ iff $L_{X} \omega=\mu \omega$ for some function $\mu$.

Let $h \in G(M, \omega)_{0}$ and $H=\left\{h_{t}\right\}_{t \in I}$ be an isotopy such that $h_{1}=h$, $h_{0}=\mathrm{id}$, and each $h_{t}$ stabilizes outside a fixed compact $K \subset M$. Of course any contact isotopy determines a family of contact vector fields, namely,

$$
X_{t}(t)=\frac{d h_{t}}{d t}\left(h_{t}^{-1}(x)\right)
$$

In fact, one has $L_{X_{t}} \omega=\mu_{t} \omega$ with $\mu_{t}=\left(d / d t\left(\ln \lambda_{t}\right)\right) h_{t}^{-1}$ where $h_{t}^{*} \omega=\lambda_{t} \omega$.

Let $Z$ denote a unique vector field satisfying $i(Z) \omega=1$ and $i(Z) d \omega=0$. A contact vector field $X$ is called horizontal if $i(X) \omega=0$. A dual concept is a semibasic form, i.e., any 1-form $\alpha$ such that $\alpha(Z)=0$, and the duality is established by the isomorphism $d \omega: X \mapsto i(X) d \omega$ : It is of particular importance and specific for $\mathscr{X}_{\omega}(M)$ among other classical Lie algebras of infinitesimal automorphisms that the following holds.

Proposition 4. There is an isomorphism $\tilde{\omega}: \mathscr{X}_{\omega}(M) \rightarrow C^{\infty}(M)$ by $\tilde{\omega}(X)=$ $i(X) \omega$. For $f \in C^{\infty}(M)$ we have $\tilde{\omega}^{-1}(f)=f Z+(d \omega)^{-1}((i(Z) d f) \omega-d f)$.

As a consequence we have the following partition properties of contact automorphisms. 
Corollary 3. Let $X$ be a compactly supported element of $\mathscr{Z}_{\omega}(M)$ and $M=$ $\bigcup_{i=1}^{r} U_{i}$ a finite open cover of $M$. Then there is a decomposition $X=X_{1}+\cdots+X_{r}$ such that $X_{i} \in \chi_{\omega}(M)$ and $\operatorname{supp}\left(X_{i}\right) \subset U_{i}$.

It is easily seen by making use of a partition of unity.

Corollary 4. Let $h \in G(M, \omega)_{0}$ and $M=\bigcup_{i=1}^{r} U_{i}$ be a finite open cover of $M$. Then there exists a decomposition $H=h_{s} \cdots h_{1}$ with $h_{k} \in G(M, \omega)_{0}$ and such that $\operatorname{supp}\left(h_{k}\right) \subset U_{i(k)}$ for $k=1, \ldots, s$.

Proof. Suppose $h \in G(M, \omega)_{0}$, and let $X=X_{1}$ be a compactly supported contact vector field induced uniquely by a compactly supported isotopy $\left\{h_{t}\right\}$ connecting $h$ with the identity. We then have $\exp (X)=h$.

It is well known that there is a finite open cover $\left\{V_{k}\right\}_{k=1}^{s}$ of $\operatorname{supp}(h)=$ $\operatorname{supp}(X)$ such that the cover $\left\{V_{k} \cup V_{l}\right\}_{(k, l)}$, where $(k, l)$ runs over all pairs such that $V_{k} \cap V_{l} \neq \varnothing$, is finer than $\left\{U_{i}\right\}$. Due to Corollary 3 we have a decomposition $X=Y_{1}+\cdots+Y_{s}$ where $Y_{k} \in \mathscr{L}_{\omega}(M)$ and $\operatorname{supp}\left(Y_{k}\right) \subset V_{k}$. Let $Z_{k}=Y_{1}+\cdots+Y_{k}$ and $f_{k}=\exp \left(Z_{k}\right), k=1, \ldots, s$. We define $g_{0}=$ id and for $k \geq 1, g_{k}=f_{k}$ whenever $V_{k} \cap V_{k+1} \neq \varnothing$, and $g_{k}=$ id otherwise. Then we set $h_{k}=g_{k} g_{k-1}^{-1}$. Thus we have $h=f_{s}=h_{s} \cdots h_{1}$ and $\operatorname{supp}\left(h_{k}\right) \subset U_{i(k)}$, as required.

Now we are in a position to solve in the affirmative the problem posed by Banyaga in [3].

Theorem 2. Let $\left(M_{i}, \omega_{i}\right), i=1,2$, be a contact manifold and $G\left(M_{i}, \omega_{i}\right)$ the group of its contact automorphisms. If there exists a group isomorphism $\Phi: G\left(M_{1}, \omega_{1}\right) \rightarrow G\left(M_{2}, \omega_{2}\right)$, then there exists a contact diffeomorphism $\phi:$ $M_{1} \rightarrow M_{2}$ such that $\Phi(f)=\phi f \phi^{-1}$ for any $f \in G\left(M_{1}, \omega_{1}\right)$.

Proof. Axiom 1 is satisfied by Corollary 4. We show that Axiom 2 holds for $G(M, \omega)$. In fact, by Darboux theorem at any $p \in M$ there is a local coordinate system $\left(U, x^{0}, x^{1}, \ldots, x^{2 n}\right)$ such that $\omega=d x^{0}+\sum_{i=1}^{n} x^{n+i} d x^{i}$ on $U$. Some suitable extensions of $\partial_{0}, \partial_{i}, \partial_{n+i}-x^{i} \partial_{0}, i=1, \ldots, n$, belong to $\mathscr{L}_{\omega}(M)$, and the first part of the axiom follows easily by Proposition 4. Moreover, suitable extensions of the vector fields

$$
-x^{n+i} \partial_{i}+\frac{1}{2}\left(x^{n+i}\right)^{2} \partial_{0}, \quad-x^{i} \partial_{n+i}+\frac{1}{2}\left(x^{i}\right)^{2} \partial_{0}, \quad i=1, \ldots, n,
$$

are also elements of $\mathscr{X}_{\omega}(M)$ (see [9, p. 135]), and so does some extension $X$ of their sum

$$
\sum_{i}-x^{i} \partial_{n+i}-x^{n+i} \partial_{i}+\frac{1}{2}\left(\left(x^{i}\right)^{2}+\left(x^{n+i}\right)^{2}\right) \partial_{0}
$$

In order to prove the second part of the axiom let $W$ be an open ball with the center at $x=0$, where $\bar{W} \subset U$, and we have to find $f \in G(M, \omega)$ such that $\operatorname{Fix}(f)=(M-W) \cup\{0\}$. Take $k \in C^{\infty}(M)$ such that $k(x) \neq 0$ for $x \in W-\{0\}$ and $k(x)=0$ otherwise and such that $d k(0)=0$. Then, in view of Proposition 4, $\tilde{X}=\tilde{\omega}^{-1}(k \tilde{\omega}(X))$ is in $G(M, \omega)$, and $\exp (\tilde{X})$ satisfies the claim.

Axiom 3 is also satisfied as $G(M, \omega)$ fulfills the path transitivity (again by making use of Proposition 4). Finally, the fact that $\phi$ is a contact diffeomorphism follows from a Pursell-Shanks type theorem for contact structures proved by Omori in [9]. 


\section{REFERENCES}

1. A. Banyaga, Sur la structure du groupe des difféomorphismes qui préservent une forme symplectique, Comment. Math. Helv. 53 (1978), 174-227.

2. __ On isomorphic classical diffeomorphism groups. I, Proc. Amer. Math. Soc. 98 (1985), 113-118.

3. __ On isomorphic classical diffeomorphism groups. II, J. Differential Geom. 28 (1988), 23-35.

4. A. Banyaga, R. de la Llave, and C. E. Wayne, Cohomology equations and commutators of germs of contact diffeomorphisms, Trans. Amer. Math. Soc. 312 (1989), 755-778.

5. R. P. Filipkiewicz, Isomorphism between diffeomorphism groups, Ergodic Theory Dynamical Systems 2 (1982), 159-171.

6. J. Gray, Some global properties of contact structures, Ann. of Math. (2) 69 (1959), 421-450.

7. A. Koriyama, Y. Maeda, and H. Omori, On Lie algebras of vector fields, Trans. Amer. Math. Soc. 226 (1977), 89-119.

8. J. Mather, Commutators of diffeomorphisms. I, II, Comment. Math. Helv. 49 (1974), 512-558; 50 (1975), 33-40.

9. H. Omori, Infinite dimensional Lie transformation groups, Lecture Notes in Math., vol. 427, Springer-Verlag, New York, 1974.

10. J. Palis and S. Smale, Structural stability theorems, Proc. Sympos. Pure Math., vol. 14, Amer. Math. Soc., Providence, RI, 1970, pp. 223-231.

11. L. E. Pursell and M. E. Shanks, The Lie algebra of a smooth manifold, Proc. Amer. Math. Soc. 5 (1954), 468-472.

12. T. Rybicki, On nontransitive groups of diffeomorphisms, preprint.

13. F. Takens, Characterization of a differentiable structure by its group of diffeomorphisms, Bol. Soc. Brasil Math. 10 (1979), 17-25.

14. W. Thurston, Foliations and groups of diffeomorphisms, Bull. Amer. Math. Soc. 80 (1974), 304-307.

15. J. V. Whittaker, On isomorphic groups and homeomorphic spaces, Ann. of Math. (2) 78 (1963), 74-91.

Institute of Mathematics, Pedagogical University, ul. Rejtana 16A, 35-310 Rzeszow, Poland 